\title{
New Constructions and Applications of Trapdoor DDH Groups
}

\author{
Yannick Seurin \\ ANSSI, Paris, France \\ yannick. seurin@m $4 \mathrm{x}$. org
}

\begin{abstract}
Trapdoor Decisional Diffie-Hellman (TDDH) groups, introduced by Dent and Galbraith (ANTS 2006), are groups where the DDH problem is hard, unless one is in possession of a secret trapdoor which enables solving it efficiently. Despite their intuitively appealing properties, they have found up to now very few cryptographic applications. Moreover, among the two constructions of such groups proposed by Dent and Galbraith, only a single one based on hidden pairings remains unbroken. In this paper, we extend the set of trapdoor DDH groups by giving a construction based on composite residuosity. We also introduce a more restrictive variant of these groups that we name static trapdoor $\mathrm{DDH}$ groups, where the trapdoor only enables to solve the DDH problem with respect to a fixed pair $\left(G, G^{x}\right)$ of group elements. We give two constructions for such groups whose security relies respectively on the RSA and the factoring assumptions. Then, we show that static trapdoor DDH groups yield elementary constructions of convertible undeniable signature schemes allowing delegatable verification. Using our constructions of static trapdoor DDH groups from the RSA or the factoring assumption, we obtain slightly simpler variants of the undeniable signature schemes of respectively Gennaro, Rabin, and Krawczyk (J. Cryptology, 2000) and Galbraith and Mao (CT-RSA 2003). These new schemes are conceptually more satisfying since they can strictly be viewed as instantiations, in an adequate group, of the original undeniable signature scheme of Chaum and van Antwerpen (CRYPTO '89).
\end{abstract}

\section{Introduction}

The CDH and DDH Problems. Given a group $\mathbb{G}$ and an element $G \in \mathbb{G}$ of large order, the Computational Diffie-Hellman (CDH) problem is to compute $G^{x y}$, given $G^{x}$ and $G^{y}$ for random integers $x, y$. The Decisional DiffieHellman (DDH) problem is to distinguish the two distributions $\left(G^{x}, G^{y}, G^{x y}\right)$ and $\left(G^{x}, G^{y}, G^{z}\right)$ for random and independent integers $x, y, z$. Usually, when considering the status of various groups with respect to the $\mathrm{CDH}$ and DDH problems, one of the following two cases arises: either the CDH and DDH problems are both presumably hard (this is the case for example for subgroups of large prime order of $\mathbb{Z}_{p}^{*}, p$ prime), or the group is a so-called gap group: the $\mathrm{CDH}$ problem is (presumably) hard while the DDH problem is universally easy (i.e. easy given only the description of the group law, which seems to be the

K. Kurosawa and G. Hanaoka (Eds.): PKC 2013, LNCS 7778, pp. 443-460, 2013.

(C) International Association for Cryptologic Research 2013 
minimal publicly available information to obtain useful applications). The latter case typically arises in certain elliptic curve groups equipped with bilinear pairings [3522], and has given rise to many important applications in cryptography 3234 .

Trapdoor DDH Groups. Trapdoor DDH groups (TDDH groups for short), introduced by Dent and Galbraith [18, lie somewhere between the above two cases. These are groups where the DDH problem is hard, except if one possesses a trapdoor for solving it efficiently. Dent and Galbraith gave two candidates for such groups based on the concept of hidden pairings, one in elliptic curves over the ring $\mathbb{Z}_{N}$, where $N$ is hard to factor, and the other one based on Frey's idea of disguising an elliptic curve [21]. Subsequently, the second proposal was broken by Morales [38. Since the DDH problem is the basis of so many cryptosystems [2], the concept of trapdoor DDH groups is very attractive. Indeed, it should enable to control more precisely who is able to solve the DDH problem in a system. This may help in situations where there is a conflict between security, which requires a group where the DDH problem is hard, and some interesting additional functionalities that could be achieved thanks to an algorithm for solving the DDH problem. One example that comes to mind is threshold ElGamal encryption. In threshold ElGamal encryption [19, given a secret/public key pair $\left(x, X=G^{x}\right)$, each decryption server is given a share $x_{i}$ of the secret key, to which is associated a "partial" public key $G^{x_{i}}$. In order to decrypt a ciphertext $(R, Y)=\left(G^{r}, M X^{r}\right)$, each server participating to decryption must compute a decryption share $S_{i}=$ $R^{x_{i}}$. Hence, checking whether a decryption share from a server is correct or not amounts to deciding whether $\left(X_{i}, R, S_{i}\right)$ is a DDH tuple or not. Yet INDCPA-security of ElGamal encryption is equivalent to the hardness of the DDH problem in the underlying group $\mathbb{G}[46$. Hence, there seems to be no other choice than using a group where the DDH problem is hard, thereby condemning other participants to be unable to distinguish correct decryption shares from incorrect ones. We do not claim that TDDH groups are the best way to solve this problem (this can be more easily achieved by having each server provide a non-interactive zero-knowledge proof that his decryption share is correctly computed), and this example only serves to argue that sometimes, one may want that only some authorized party be able to solve the DDH problem. Despite these considerations, TDDH groups have found up to now very few cryptographic applications. In their original paper, Dent and Galbraith gave only one example, namely an identification scheme. To the best of our knowledge, the only previous paper proposing a non-trivial application of TDDH groups (namely the construction of statistically hiding sets, a variant of zero-knowledge sets) is due to Prabhakaran and Xue 43 .

Contributions of This Work. The contributions of this paper can be summarized as follows. First, at a conceptual level, we refine the definition of TDDH groups of Dent and Galbraith by requiring that the $\mathrm{CDH}$ problem remain hard even given the trapdoor for solving the DDH problem. This was not made explicit in the formalization by Dent and Galbraith, yet we think that this is probably 
a key feature for many interesting applications, such as undeniable signatures for example. We also broaden the set of constructions of trapdoor DDH groups. We propose a new construction based on composite residuosity in $\mathbb{Z}_{N^{2}}^{*}$ (similar considerations have been made by [6], albeit not in the formalism of TDDH groups), and identify under which hardness assumptions this group satisfies our definition. A drawback of this construction is that it lacks what we call perfect soundness, meaning that the algorithm solving the DDH problem with the trapdoor can sometimes err and declare valid a non-DH tuple.

Then, we introduce a variant of trapdoor DDH groups that we name static trapdoor DDH groups. Their definition is very similar to the one of trapdoor $\mathrm{DDH}$ groups, except that the trapdoor for solving the DDH problem is now dedicated to a specific pair of group elements $\left(G, G^{x}\right)$, hence the name static. We then show that such groups can be easily constructed from the RSA and the factoring problems. This concept abstracts some of the ideas underlying the work of Hofheinz and Kiltz [31, who showed that the Strong Diffie-Hellman (SDH) problem (i.e. solving the $\mathrm{CDH}$ problem given access to a static DDH oracle) is hard in the so-called group of signed quadratic residues under the factoring assumption.

Finally, we describe a very natural application of (static or not) TDDH groups to convertible undeniable signature schemes. Namely, the construction we propose is exactly the original undeniable signature scheme proposed by Chaum and van Antwerpen [12] (for which deciding the validity of a signature is equivalent to solving the DDH problem), but in a TDDH group rather than simply a group where the DDH problem is hard. The trapdoor for solving the DDH problem can then be used to universally convert or delegate verification of signatures. Once instantiated with our proposals of static TDDH groups based on the RSA or the factoring problems, we obtain schemes similar to previous RSA-based undeniable signature schemes due to Gennaro, Rabin, and Krawczyk [26] and Galbraith and Mao [23. However, these new schemes are conceptually simpler and easier to analyze. Moreover, since they are strict instantiations of the Chaum and van Antwerpen scheme, their confirmation and disavowal protocols can use classical proofs of equality or inequality of discrete logarithms, which are simpler and more efficient than what was proposed previously for the schemes of [26 23].

Open Problems. Two key features of TDDH groups are perfect soundness (the property that the algorithm for solving the DDH problem with the trapdoor perfectly distinguishes $\mathrm{DH}$ tuples from non-DH tuples), and the possibility to securely hash into the group (see discussion in Section 2.3). However, none of the two candidates for TDDH groups (the hidden pairing based proposal of [18], and our proposal in Section 3.2) fulfills both requirements. We think that providing a plausible candidate possessing both properties is the key to enable powerful applications of TDDH groups 11 A related open problem is whether there exists a (plausible construction of a) TDDH group with publicly known (ideally prime) order, since they are usually simpler to use in cryptography.

${ }^{1}$ Our examples of static TDDH groups do fulfill both requirement, however non-static TDDH groups would allow more flexibility in cryptographic applications. 
Organization. In Section 2 we give some basic definitions and introduce some of the tools we will need in the remainder of the paper. In Section 3, we define trapdoor DDH groups, and give a construction based on composite residuosity. In Section 4 we introduce static trapdoor DDH groups, and give two constructions based on respectively the RSA and the factoring assumptions. Finally, in Section [5] we show how to obtain convertible undeniable signature schemes from static TDDH groups, and discuss their instantiation with the constructions described previously.

\section{Preliminaries}

\subsection{Notation and Definitions}

The set of integers $i$ such that $a \leq i \leq b$ will be denoted $[a ; b]$. The security parameter will be denoted $k$. A function $f$ of the security parameter is said negligible if for any $c>0, f(k) \leq 1 / k^{c}$ for sufficiently large $k$. When $S$ is a nonempty finite set, we write $s \leftarrow \$ S$ to mean that a value is sampled uniformly at random from $S$ and assigned to $s$. By $z \leftarrow \mathcal{A}^{\mathcal{O}_{1}, \mathcal{O}_{2}, \ldots}(x, y, \ldots)$ we denote the operation of running the (possibly probabilistic) algorithm $\mathcal{A}$ on inputs $x, y, \ldots$ with access to oracles $\mathcal{O}_{1}, \mathcal{O}_{2}, \ldots$ (possibly none), and letting $z$ be the output. PPT will stand for probabilistic polynomial-time. Given two Interactive Turing Machines $\mathcal{P}$ and $\mathcal{V}$, we denote $w \leftarrow\langle\mathcal{P}(x), \mathcal{V}(y)\rangle(z)$ to mean that the output of the interaction of $\mathcal{P}$ with private input $x$ and $\mathcal{V}$ with private input $y$ on common input $z$ is $w$.

Given an integer $N$, the multiplicative group of integers modulo $N$ is denoted $\mathbb{Z}_{N}^{*}$. This group has order $\phi(N)$ where $\phi(\cdot)$ is the Euler function and exponent $\lambda(N)$ where $\lambda(\cdot)$ is the Carmichael function. We denote $\mathbb{J}_{N}$ the subgroup of $\mathbb{Z}_{N}^{*}$ of all elements $x \in \mathbb{Z}_{N}^{*}$ with Jacobi symbol $\left(\frac{x}{N}\right)=1$. This subgroup has index 2 and order $\phi(N) / 2$ in $\mathbb{Z}_{N}^{*}$. Moreover it is efficiently recognizable even without the factorization of $N$ since the Jacobi symbol is efficiently computable given only $N$. We also denote $\mathbb{Q} \mathbb{R}_{N}$ the subgroup of quadratic residues of $\mathbb{Z}_{N}^{*}$. This subgroup is widely believed not to be efficiently recognizable when $N$ is composite and its factorization is unknown (this is the Quadratic Residuosity assumption). We call a prime number $p$ such that $(p-1) / 2$ is prime a safe prime.

In all the following, given a group $\mathbb{G}$, we use the notation $[\mathbb{G}]$ to denote a description of the group, i.e. an efficient algorithm for computing the group operation. This notation always implies that $\mathbb{G}$ is efficiently recognizable. We assume that it is always possible to derive from the description of the group a negligibly close upper bound on the order $|\mathbb{G}|$ of the group (in some cases the exact order may be efficiently computable), and we use the notation $|\mathbb{G}|^{+}$ to denote this upper bound 2 Given an element $G \in \mathbb{G}$, we denote ord $(G)$ its order, $\langle G\rangle$ the group generated by $G, \operatorname{Dlog}_{G}(X)$ the discrete logarithm in base

$\overline{{ }^{2} \text { E.g. when } \mathbb{G}}=\mathbb{Z}_{p}^{*}$ for some prime number $p,|\mathbb{G}|^{+}=p-1$, while when $\mathbb{G}=\mathbb{Z}_{N}^{*}$, where the factorization of $N$ is secret, $|\mathbb{G}|^{+}=N$. 
$G$ of an element $X \in\langle G\rangle$, and $\operatorname{CDH}_{G}(X, Y)=G^{\operatorname{Dlog}_{G}(X) \operatorname{Dlog}_{G}(Y)}$. We also denote $\mathcal{D H}_{G} \subset\langle G\rangle^{3}$ the set of Diffie-Hellman (DH) tuples with respect to $G$ :

$$
\mathcal{D} \mathcal{H}_{G}=\left\{\left(G^{x}, G^{y}, G^{x y}\right), x, y \in[0 ; \operatorname{ord}(G)-1]\right\}
$$

A group generator Gen is a PPT algorithm which on input a security parameter $1^{k}$, outputs a tuple $([\mathbb{G}], G, \gamma)$ where $[\mathbb{G}]$ is the description of a group $\mathbb{G}, G \in \mathbb{G}$ is an element of order $2^{\Theta(k)}$, and $\gamma$ is some arbitrary side information. We say that the $\mathrm{CDH}$ problem is hard for Gen if for any PPT adversary $\mathcal{A}$, the following probability is negligible:

$$
\begin{array}{r}
\operatorname{Pr}\left[([\mathbb{G}], G, \gamma) \leftarrow \operatorname{Gen}\left(1^{k}\right),(X, Y) \leftarrow_{\$}\langle G\rangle^{2}, Z \leftarrow \mathcal{A}([\mathbb{G}], G, \gamma ; X, Y):\right. \\
\left.Z=\mathrm{CDH}_{G}(X, Y)\right]
\end{array}
$$

We say that the DDH problem is hard for Gen if for any PPT adversary $\mathcal{A}$, the following advantage is negligible:

$$
\begin{aligned}
& \mid \operatorname{Pr}\left[([\mathbb{G}], G, \gamma) \leftarrow \operatorname{Gen}\left(1^{k}\right),(X, Y) \leftarrow \$\langle G\rangle^{2}, Z \leftarrow \mathrm{CDH}_{G}(X, Y):\right. \\
& 1 \leftarrow \mathcal{A}([\mathbb{G}], G, \gamma ; X, Y, Z)]-\operatorname{Pr}\left[([\mathbb{G}], G, \gamma) \leftarrow \operatorname{Gen}\left(1^{k}\right),(X, Y, Z) \leftarrow_{\$}\langle G\rangle^{3}:\right. \\
& 1 \leftarrow \mathcal{A}([\mathbb{G}], G, \gamma ; X, Y, Z)] \mid
\end{aligned}
$$

\subsection{Proofs of Equality and Inequality of Discrete Logarithms}

Protocols for proving, given $(G, X, Y, Z) \in \mathbb{G}$, the equality of discrete logarithms (EDL) $\operatorname{Dlog}_{G}(X)=\mathrm{D} \log _{Y}(Z)$ or the inequality of discrete logarithms (IDL) constitute (among many other applications) the heart of respectively the confirmation and disavowal protocols for many undeniable signature schemes, and have therefore been the subject of many works. They vary depending on the exact kind of zero-knowledge property one wants to achieve. The basic honest-verifier zero-knowledge (HVZK) proof of EDL is due to Chaum and Pedersen [11, while the simplest HVZK proof of IDL is due to Camenish and Shoup [9]. These protocols are usually described for ambient groups $\mathbb{G}$ with publicly known prime order, in which case recognizing $\langle G\rangle$ is trivial, so that these protocols are actually proofs that a tuple $(X, Y, Z) \in \mathbb{G}^{3}$ is in $\mathcal{D} \mathcal{H}_{G}$ or not. They can be adapted to the case where the order of the ambient group is composite and secret using well-known techniques 2728 , with the caveat that if $\langle G\rangle$ is not efficiently recognizable, the verifier must be promised that $X, Y, Z \in\langle G\rangle$ since these proofs do not in general ensure membership of $X, Y, Z$ in $\langle G\rangle$ with negligible soundness 3 Stated differently, if $\mathbb{G}^{\prime}$ is a cyclic and efficiently recognizable subgroup of $\mathbb{G}$ (e.g. $\mathbb{G}=\mathbb{Z}_{N}^{*}$ and $\mathbb{G}^{\prime}=\mathbb{J}_{N}$ when $\mathbb{J}_{N}$ is cyclic), these protocols are actually proofs that a tuple $(X, Y, Z) \in \mathbb{G}^{\prime}$ is a DH tuple with respect to $G$ or not, assuming that the verifier is guaranteed that $G$ is indeed a generator of

\footnotetext{
3 The soundness of the Schnorr protocol [44], seen as a proof of membership in $\langle G\rangle$,
} is $1 / \ell$, where $\ell$ is the smallest prime factor of the order of the ambient group $\mathbb{G}$. 
$\mathbb{G}^{\prime}$ (which may not be efficiently checkable). The HVZK protocols for EDL and IDL are described in the full version of the paper [45]. They can be strengthen to achieve various notions of zero-knowledge (against cheating verifiers) using known techniques 291416 25] that we do not discuss in this paper.

The HVZK proofs of EDL and IDL can be made non-interactive in the Random Oracle Model using the Fiat-Shamir transformation [20], i.e. by having the prover compute the challenge (first message from the verifier) by itself by applying a hash function to the commitment (first message from the prover). Note that these proofs then become universally convincing.

\subsection{Hashing into Groups}

For many applications (and in particular for undeniable signatures based on the Chaum and van Antwerpen scheme [12]), it is required to securely hash into the subgroup $\langle G\rangle$ specified by the group generator Gen. We discuss this in more details in the full version of the paper [45].

\section{Trapdoor DDH Groups}

We start by defining trapdoor DDH groups. Our definition is a refinement of the one of Dent and Galbraith [18] in that we explicitly require that the CDH problem remain hard even given the trapdoor $\tau$ enabling to solve the DDH problem.

\subsection{Definition}

Definition 1. A trapdoor $\mathrm{DDH}$ group $\mathcal{T D D H}$ is a pair of algorithms (Gen, Solve) with the following properties. The trapdoor DDH group generator algorithm Gen is a PPT algorithm which takes as input a security parameter $1^{k}$ and outputs a tuple $([\mathbb{G}], G, \tau)$ where $[\mathbb{G}]$ is the description of a group $\mathbb{G}, G \in \mathbb{G}$ is a group element of order $2^{\Theta(k)}$, and $\tau$ is a trapdoor information, such that:

i) hardness of $\mathrm{DDH}$ without the trapdoor: the DDH problem is hard for the group generator $\mathrm{Gen}^{\prime}$ which outputs only $([\mathbb{G}], G)$;

ii) hardness of $\mathrm{CDH}$ with the trapdoor: the $C D H$ problem is hard for Gen.

Solve is a deterministic polynomial-time algorithm which takes as input $([\mathbb{G}], G, \tau)$ and a tuple $(X, Y, Z) \in \mathbb{G}^{3}$, either accepts (outputs 1 ) or rejects (outputs 0$)$, and satisfies the following:

iii) completeness: for all $([\mathbb{G}], G, \tau)$ possibly output by Gen, Solve always accepts on input a DH tuple $(X, Y, Z) \in \mathcal{D H}_{G}$;

iv) soundness: for any PPT adversary $\mathcal{A}$, the following probability is negligible:

$$
\begin{aligned}
\operatorname{Pr}\left[([\mathbb{G}], G, \tau) \leftarrow \operatorname{Gen}\left(1^{k}\right),(X, Y) \leftarrow \$\langle G\rangle^{2}, Z \leftarrow \mathcal{A}([\mathbb{G}], G ; X, Y):\right. \\
\left.1 \leftarrow \operatorname{Solve}([\mathbb{G}], G, \tau ; X, Y, Z) \wedge(X, Y, Z) \notin \mathcal{D} \mathcal{H}_{G}\right] .
\end{aligned}
$$


We say that $\mathcal{T} \mathcal{D D} \mathcal{H}$ has perfect soundness when Solve always rejects on input a non-DH tuple $(X, Y, Z)$, so that the above probability is zero.

Note that the soundness condition implies in particular that Solve, on input a uniformly random tuple $(X, Y, Z) \in \mathbb{G}^{3}$, accepts only with negligible probability. We silently assumed in the above definition that Solve is always run with a correctly generated trapdoor. This is safe for all examples presented below since there is an efficient way, given $([\mathbb{G}], G, \tau)$, to check whether the trapdoor is correct. We assume that Solve outputs a special symbol $\perp$ when this is not the case. We recall the original proposal of a TDDH group based on hidden pairings by Dent and Galbraith [18] in the full version of the paper [45].

\subsection{A TDDH Group Based on Composite Residuosity}

In this section, we describe a TDDH group $\mathcal{T} \mathcal{D} \mathcal{D} \mathcal{H}_{\mathrm{BCP}}$ based on the group of quadratic residues modulo $N^{2}$, where $N$ is an RSA modulus. This group was first considered by Bresson, Catalano, and Pointcheval [6], who noticed that when the factorization of $N$ is publicly available, this constitutes a gap group, i.e. a group where the $\mathrm{CDH}$ problem is hard and the DDH problem is easy. Here, we show that it constitutes in fact a TDDH group when the factorization of $N$ is kept secret and used as the trapdoor.

We first recall some basic facts about the group of quadratic residues modulo $N^{2}$, where $N$ is an RSA modulus. Let $p, q$ be two safe primes where $p=2 p^{\prime}+1$ and $q=2 q^{\prime}+1$ ( $p^{\prime}$ and $q^{\prime}$ primes), and $N=p q$. The group $\mathbb{Q R}_{N^{2}}$ of quadratic residues modulo $N^{2}$ is a cyclic group of order $m=N p^{\prime} q^{\prime}$. We define the notion of partial discrete logarithm.

Definition 2 (Partial Discrete Logarithm). Given a generator $G$ of $\mathbb{Q R}_{N^{2}}$, the partial discrete logarithm of a group element $X \in \mathbb{Q R}_{N^{2}}$ is defined as $\mathrm{PDlog} \log _{G}(X)=\mathrm{Dlog}_{G}(X) \bmod N$.

Computing the partial discrete logarithm is believed to be hard without the factorization of $N 4$ However, it can be efficiently computed given the prime factors of $N$ (or simply $\lambda(N)$ ) as follows [42]:

1. input: $N, \lambda(N)$, generator $G$ of $\mathbb{Q R}_{N^{2}}$ and $X \in \mathbb{Q R}_{N^{2}} ;$ output: $\operatorname{PDlog}_{G}(X)$

2. for integers $u \in\left[0 ; N^{2}-1\right]$ such that $u=1 \bmod N$, define the function (having integer values) $\mathcal{L}(u)=(u-1) / N$

3. return

$$
\frac{\mathcal{L}\left(X^{\lambda(N)} \bmod N^{2}\right)}{\mathcal{L}\left(G^{\lambda(N)} \bmod N^{2}\right)} \bmod N .
$$

We now formally describe the TDDH group $\mathcal{T} \mathcal{D} \mathcal{D} \mathcal{H}_{\mathrm{BCP}}$. On input the security

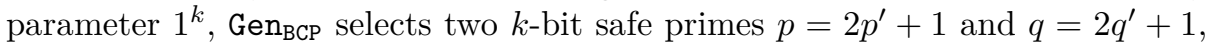

\footnotetext{
${ }^{4}$ As noted by Paillier [42] and in [6], the Partial Discrete Logarithm problem can be shown equivalent to the Composite Residuosity Class problem in the particular case considered here.
} 
sets $N=p q$, selects a random generator $G$ of $\mathbb{Q R}_{N^{2}}$, and outputs $\left(\left[\mathbb{Z}_{N^{2}}^{*}\right], G, \tau=\right.$ $(p, q))$. The Solve $\mathrm{BCP}$ algorithm works as follows: on input a tuple $(X, Y, Z) \in$ $\left(\mathbb{Z}_{N^{2}}^{*}\right)^{3}$ (as well as the trapdoor $\tau=(p, q)$ ), it checks whether $X, Y, Z \in \mathbb{Q R}_{N^{2}}$, computes $x^{\prime}=\mathrm{PDlog}_{G}(X), y^{\prime}=\mathrm{PDlog}_{G}(Y)$, and $z^{\prime}=\mathrm{PDlog}_{G}(Z)$ as described above, and checks whether $z^{\prime}=x^{\prime} y^{\prime} \bmod N$. It accepts if this holds and rejects otherwise. The security of this TDDH group relies on a "partial" version of the $\mathrm{CDH}$ problem, defined as follows.

Definition 3 (Partial CDH Problem). We say that the Partial CDH problem is hard if for any PPT algorithm $\mathcal{A}$, the following probability is negligible:

$$
\begin{array}{r}
\operatorname{Pr}\left[\left(\left[\mathbb{Z}_{N^{2}}^{*}\right], G, \tau\right) \leftarrow \operatorname{Gen}_{\mathrm{BCP}}\left(1^{k}\right),(X, Y) \leftarrow \$\langle G\rangle^{2}, Z \leftarrow \mathcal{A}\left(\left[\mathbb{Z}_{N^{2}}^{*}\right], G ; X, Y\right):\right. \\
\left.\operatorname{Dlog}_{G}(Z) \equiv \operatorname{Dlog}_{G}(X) \operatorname{Dlog}_{G}(Y) \bmod N\right]
\end{array}
$$

Theorem 1. Assuming that the DDH problem (without the factorization of $N$ ), the $C D H$ problem (with the factorization of $N$ ), and the Partial $C D H$ problem (without the factorization of $N$ ) are hard for $\mathbb{Q R}_{N^{2}}, \mathcal{T} \mathcal{D} \mathcal{D} \mathcal{H}_{\mathrm{BCP}}$ is a trapdoor DDH group.

Proof. We prove that properties $i$ ) to $i v$ ) of Definition 1 are satisfied. Properties $i$ ) and $i i$ ) follow directly from the assumptions that respectively the DDH (without the factorization of $N$ ) and the $\mathrm{CDH}$ (with the factorization of $N$ ) problems are hard in $\mathbb{Q} \mathbb{R}_{N^{2}}$. Property iii) is straightforward to verify by defini-

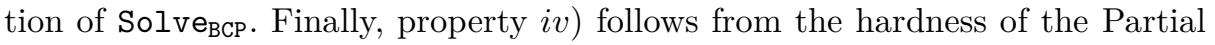
$\mathrm{CDH}$ problem.

Note that this TDDH group does not have perfect soundness. In particular, on input a random tuple $(X, Y, Z) \in\left(\mathbb{Q R}_{N^{2}}\right)^{3}$, there is a negligible probability that

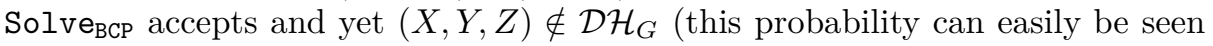
to be $\mathcal{O}(1 / N)[6])$. Moreover, given the trapdoor $\tau=(p, q)$, and two random elements $(X, Y) \in\left(\mathbb{Q R}_{N^{2}}\right)^{2}$, it is easy to generate $Z$ such that $(X, Y, Z) \notin \mathcal{D} \mathcal{H}_{G}$ and yet Solve $\mathrm{BCP}_{\mathrm{BC}}$ accepts on input $(X, Y, Z)$ : simply compute $x^{\prime}=\mathrm{PDlog}_{G}(X)$ and $y^{\prime}=\operatorname{PDlog}_{G}(Y)$ and output $G^{x^{\prime} y^{\prime} \bmod N}$. Alternatively, given two random elements $(X, Y) \in\left(\mathbb{Q R}_{N^{2}}\right)^{2}$ and $Z=\operatorname{CDH}_{G}(X, Y)$, it is easy to compute $Z^{\prime} \neq Z$

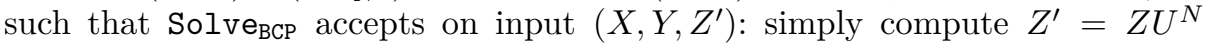
for some random $U \in \mathbb{Q R}_{N^{2}}$. This may be of concern in some applications, especially for undeniable signature schemes where Solve is typically used to check the validity of signatures (see Section 5 ) 5

\section{Static Trapdoor DDH Groups}

In this section, we define and construct static trapdoor DDH groups. They are similar to trapdoor DDH groups as defined in Section 3. except that the trapdoor only allows to solve the DDH problem with respect to a specific pair of group elements $\left(G, G^{x}\right)$.

${ }^{5}$ We note however that imperfect soundness is not a problem for the identification scheme outlined in [18]. 


\subsection{Definition}

Definition 4. A static trapdoor DDH group $\mathcal{S T D D H}$ is a tuple of algorithms (Gen, Samp, Solve) with the following properties. The static trapdoor DDH group generator algorithm Gen is a PPT algorithm which takes as input a security parameter $1^{k}$ and outputs a tuple $([\mathbb{G}], G, \tau)$ where $[\mathbb{G}]$ is the description of a group $\mathbb{G}, G \in \mathbb{G}$ is a group element of order $2^{\Theta(k)}$, and $\tau$ is a (master) trapdoor information, such that:

i) hardness of DDH without the trapdoor: the DDH problem is hard for the group generator $\mathrm{Gen}^{\prime}$ which outputs only $([\mathbb{G}], G)$.

Samp is a PPT algorithm which on input $([\mathbb{G}], G, \tau)$, samples uniformly at random a group element $X \leftarrow_{\$}\langle G\rangle$, and output $\oint\left(X, x, \tau_{x}\right)$ where $x=\operatorname{Dlog}_{G}(X)$ and $\tau_{x}$ is a (static) trapdoor information, such that:

ii) hardness of $\mathrm{CDH}$ with the static trapdoor: for any PPT algorithm $\mathcal{A}$, the following probability is negligible:

$$
\begin{aligned}
& \operatorname{Pr}\left[([\mathbb{G}], G, \tau) \leftarrow \operatorname{Gen}\left(1^{k}\right),\right.\left(X, x, \tau_{x}\right) \leftarrow \operatorname{Samp}([\mathbb{G}], G, \tau), Y \leftarrow \$\langle G\rangle, \\
&\left.Z \leftarrow \mathcal{A}\left([\mathbb{G}], G ; X, Y ; \tau_{x}\right): Z=\mathrm{CDH}_{G}(X, Y)\right] .
\end{aligned}
$$

Solve is a deterministic polynomial-time algorithm which takes as input $([\mathbb{G}], G)$, a tuple $(X, Y, Z) \in\langle G\rangle \times \mathbb{G}^{2}$, and the trapdoor $\tau_{x}$ for $X$, either accepts (outputs 1) or rejects (outputs 0 ), and satisfies the following:

iii) completeness: for all $([\mathbb{G}], G, \tau)$ and $\left(X, x, \tau_{x}\right)$ possibly output by Gen and Samp, and any $(Y, Z) \in \mathbb{G}^{2}$, Solve always accepts when $(X, Y, Z) \in \mathcal{D} \mathcal{H}_{G}$;

iv) soundness: for any PPT adversary $\mathcal{A}$, the following probability is negligible:

$$
\begin{gathered}
\operatorname{Pr}\left[([\mathbb{G}], G, \tau) \leftarrow \operatorname{Gen}\left(1^{k}\right),\left(X, x, \tau_{x}\right) \leftarrow \operatorname{Samp}([\mathbb{G}], G, \tau), Y \leftarrow \$\langle G\rangle,\right. \\
\left.Z \leftarrow \mathcal{A}([\mathbb{G}], G ; X, Y): 1 \leftarrow \operatorname{Solve}\left([\mathbb{G}], G ; X, Y, Z ; \tau_{x}\right) \wedge(X, Y, Z) \notin \mathcal{D} \mathcal{H}_{G}\right]
\end{gathered}
$$

We say that $\mathcal{S} \mathcal{T} \mathcal{D} \mathcal{D} \mathcal{H}$ has perfect soundness when Solve always rejects on input a non-DH tuple $(X, Y, Z)$, so that the above probability is zero.

Again, we silently assumed that Solve is always run with the correct trapdoor $\tau_{x}$ because in all examples below this can be checked efficiently. In the remainder of this section, we propose two constructions of static TDDH groups based respectively on the RSA problem and the factoring problem.

\subsection{A Construction Based on the RSA Problem}

We first show how a static TDDH group can be obtained from the RSA problem. Let $N=p q$ be an RSA modulus. When $(p-1) / 2$ and $(q-1) / 2$ are coprime,

\footnotetext{
${ }^{6}$ We stress that in typical applications, $x$ is retained by an authorized user and is never made available to the adversary.
} 
then the subgroup $\mathbb{J}_{N}$ of $\mathbb{Z}_{N}^{*}$ is cyclic. Moreover, when $p$ and $q$ are distinct safe primes, the DDH problem is widely believed to be hard in $\mathbb{J}_{N}[2]$. We define the static TDDH group $\mathcal{S} \mathcal{T} \mathcal{D} \mathcal{D} \mathcal{H}_{\mathrm{RSA}}$ as follows. On input $1^{k}$, the group generator Gen $_{\mathrm{RSA}}$ selects two $k$-bit safe primes $p=2 p^{\prime}+1$ and $q=2 q^{\prime}+1$, defines $N=p q$ and $m=(p-1)(q-1) / 2=2 p^{\prime} q^{\prime}$, selects a generator $G$ of $\mathbb{J}_{N}$, and outputs $\left(\left[\mathbb{J}_{N}\right], G, \tau=m\right)$. The $\operatorname{Samp}_{\mathrm{RSA}}$ algorithm, on input $\left(\left[\mathbb{J}_{N}\right], G, m\right)$, draws a random $x \leftarrow \$ \mathbb{Z}_{m}^{*}$, computes $X=G^{x}, \tau_{x}=1 / x \bmod m$, and outputs $\left(X, x, \tau_{x}\right)$ (note that we slightly deviate from Definition 4 here since $X$ is not uniformly random in $\langle G\rangle$, but the statistical distance is negligible). Algorithm Solve $\mathrm{RSA}_{\mathrm{RA}}$, on input $\left(\left[\mathbb{J}_{N}\right], G ; X, Y, Z ; \tau_{x}\right)$, first checks that $X, Y, Z \in \mathbb{J}_{N}$, that the trapdoor is correct by verifying whether $X^{\tau_{x}}=G$ (it outputs $\perp$ if this does not hold), and outputs 1 iff $Z^{\tau_{x}}=Y$.

Definition 5. We say that the RSA problem is hard for $\mathbb{J}_{N}$ if for any PPT adversary $\mathcal{A}$, the following probability is negligible:

$$
\operatorname{Pr}\left[\left(\left[\mathbb{J}_{N}\right], G, m\right) \leftarrow \operatorname{Gen}_{\mathrm{RSA}}\left(1^{k}\right), e \leftarrow \$ \mathbb{Z}_{m}^{*}, Y \leftarrow \leftarrow_{\$} \mathbb{J}_{N}, Z \leftarrow \mathcal{A}\left(\left[\mathbb{J}_{N}\right], Y, e\right): Z^{e}=Y\right]
$$

Theorem 2. Assuming that the DDH problem and the RSA problem are hard in $\mathbb{J}_{N}$ (for $N$ the product of two distinct safe primes), $\mathcal{S T D D} \mathcal{H}_{\mathrm{RSA}}$ is a static TDDH group with perfect soundness.

Proof. We show that properties $i$ ) to $i v$ ) of Definition 4 hold. Property $i$ ) holds by assumption that DDH is hard for $\mathbb{J}_{N}$. We now prove property $i i$ ). Assume that there is an adversary $\mathcal{A}$ breaking property $i$ ). We construct a reduction $\mathcal{R}$ that solves the RSA problem as follows. The reduction is given the product $N=p q$ of two safe primes, a random $e$ coprime with $m=(p-1)(q-1) / 2$, and a random challenge $Y \in \mathbb{J}_{N}$ of which it must compute the $e$-th root. The reduction draws a random $X \leftarrow \leftarrow_{\$} \mathbb{J}_{N}$. With overwhelming probability, $X$ is a generator of $\mathbb{J}_{N}$ since $p$ and $q$ are safe primes. The reduction defines $G=X^{e}$, and runs $\mathcal{A}$ on input $\left(\left[\mathbb{J}_{N}\right], G ; X, Y ; e\right)$. The statistical distance between inputs $(G, X, Y)$ in the simulated experiment and in the real $\mathrm{CDH}$ experiment defining property $i i$ ) is negligible (the difference coming from cases where $X$ does not generate $\mathbb{J}_{N}$ ). Moreover, $e$ is the correct trapdoor for $X$ since $G=X^{e}$ implies $e=1 / x \bmod m$, where $x=\operatorname{Dlog}_{G}(X)$. Hence, $\mathcal{A}$ returns the correct value $Z=\operatorname{CDH}_{G}(X, Y)$ with probability negligibly close to its advantage, in which case $Z=Y^{x}$, which implies $Z^{e}=Y$, so that $Z$ is indeed the $e$-th root of $Y$. The running time of $\mathcal{R}$ is similar to the one of $\mathcal{A}$ and its success probability is negligibly close to the one of $\mathcal{A}$. Property iii) is clear, and $\mathcal{S} \mathcal{T} \mathcal{D} \mathcal{D} \mathcal{H}_{\mathrm{RSA}}$ has perfect soundness since by definition of $\operatorname{Samp}_{\mathrm{RSA}}, x$ is coprime to $m$ so that $Z^{\tau_{x}}=Y \Leftrightarrow Z^{x \tau_{x}}=Y^{x} \Leftrightarrow Z=Y^{x}$.

\subsection{A Construction Based on Signed Quadratic Residues}

In this section, we describe a static TDDH group based on signed quadratic residues, whose usefulness for cryptography was first noticed by Hofheinz and Kiltz 31. This can be seen as a variant of $\mathcal{S} \mathcal{T} \mathcal{D} \mathcal{D} \mathcal{H}_{\mathrm{RSA}}$ described above, whose security relies on the factoring problem rather than the RSA problem. We first give some definitions. 
Definition 6. Let $N$ be an odd positive integer such that $-1 \in \mathbb{J}_{N}$. We denote $\mathbb{J}_{N}^{+}$the quotient group $\mathbb{J}_{N} /\{-1,1\}$. We identify $\mathbb{J}_{N}^{+}$with the set $\mathbb{J}_{N} \cap[1 ;(N-1) / 2]$ equipped with the group operation $\circ$ defined as $a \circ b=|a b \bmod N|$, where $\mid x \bmod$ $N \mid$ is defined as the absolute value of $x \bmod N$ when representing elements of $\mathbb{Z}_{N}$ as integers in $[-(N-1) / 2 ;(N-1) / 2]$.

To be completely rigorous, the mapping which to an element $\{-x, x\} \in \mathbb{J}_{N}^{+}$ associates $|x|$ is a group isomorphism between $\mathbb{J}_{N}^{+}$and $\left(\mathbb{J}_{N} \cap[1,(N-1) / 2], \circ\right)$.

Let $N=p q$ be a Blum integer (i.e. $p$ and $q$ are two primes such that $p \equiv q \equiv$ $3 \bmod 4)$. Then $-1 \in \mathbb{J}_{N}$ so that we can define $\mathbb{J}_{N}^{+}$, which in this particular case is named the group of signed quadratic residues and denoted $\mathbb{Q} \mathbb{R}_{N}^{+} \sqrt{7}$ Its order is $\phi(N) / 4=(p-1)(q-1) / 4$. The most interesting points to notice about this group is that it is efficiently recognizable (since it is isomorphic to $\mathbb{J}_{N} \cap[1 ;(N-1) / 2]$ ), and that the squaring operation is one-to-one so that any $x \in \mathbb{Q R}_{N}^{+}$has a unique square root in $\mathbb{Q R}_{N}^{+}$(more precisely, for any $x \in \mathbb{Q R}_{N}^{+}$, either $x$ or $-x \bmod N$ is a quadratic residue $\bmod N$, and exactly one corresponding square root is in $\left.\mathbb{Q R}_{N}^{+}\right)$. Moreover, when $(p-1) / 2$ and $(q-1) / 2$ are coprime, then $\mathbb{J}_{N}$ is cyclic and so is $\mathbb{Q R}_{N}^{+}$. See [31] for proofs of these basic facts.

In the following, we restrict ourselves for simplicity to the special case where $N$ is the product of two distinct safe primes. This implies that $N$ is a Blum integer, and that $(p-1) / 2$ and $(q-1) / 2$ are coprime so that $\mathbb{Q R}_{N}^{+}$is cyclic. Moreover, a uniformly random element of $\mathbb{Q} \mathbb{R}_{N}^{+}$is a generator with overwhelming probability since the number of generators of $\mathbb{Q R}_{N}^{+}$is $\phi((p-1)(q-1) / 4)=(p-3)(q-3) / 4$.

Let $G$ be a generator of $\mathbb{Q R}_{N}^{+}$, and denote $m=\left|\mathbb{Q R}_{N}^{+}\right|=(p-1)(q-1) / 4$. Let $x \in[0 ; m-1]$ and $X=G^{x}$. To build a trapdoor enabling to solve the static DDH problem for $(G, X)$, we use the following idea: the trapdoor will be $t=2 x \pm m$ (computed over $\mathbb{Z}$ ), i.e. the value $2 x$ masked with the group order $m$. Since computing the group order $m$ is as hard as factoring $N, t$ does not reveal $x$. Now, given a group element $Y=G^{y} \in \mathbb{G}, t$ enables computing $Y^{t}=G^{2 x y}=\operatorname{CDH}_{G}(X, Y)^{2}$. This enables testing whether an element $Z$ is a correct solution to the static $\mathrm{CDH}$ problem (in other words to solve the static DDH problem) by simply checking whether $Z^{2}=Y^{t}$. However, as we will see, the static CDH problem remains as hard as computing square roots in $\mathbb{Q} \mathbb{R}_{N}^{+}$, which in turn is equivalent to factoring $N$. For what follows, we will also make the assumption that the DDH problem is hard in $\mathbb{Q R}_{N}^{+}$. The DDH problem in $\mathbb{Q} \mathbb{R}_{N}^{+}$can easily shown to be equivalent to the DDH problem in $\mathbb{J}_{N}$, which as already pointed out is widely believed to be hard when $N$ is the product of two distinct safe primes [2].

We now formally define the static TDDH group $\mathcal{S T D} \mathcal{D} \mathcal{D} \mathcal{H}_{\mathrm{SQR}}$. For ease of exposition, given an odd integer $m$, we define the function $\xi$ from $[0 ; m-1]$ to $\{1,3,5, \ldots, 2 m-3,2 m-1\}$ as:

$$
\left\{\begin{array}{l}
\xi(x)=2 x+m \text { if } x \in[0 ;(m-1) / 2] \\
\xi(x)=2 x-m \text { if } x \in[(m+1) / 2 ; m-1] .
\end{array}\right.
$$

\footnotetext{
$\overline{{ }^{7} \text { We warn that }} \mathbb{Q R}_{N}^{+}$is not equal to $\mathbb{Q R}_{N} /\{-1,1\}$ for the good reason that $-1 \notin \mathbb{Q R}_{N}$
} when $N$ is a Blum integer. 
$\xi(x)$ is the unique odd integer $t \in[1 ; 2 m-1]$ such that $t=2 x \pm m$.

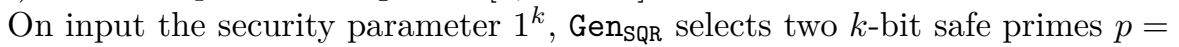
$2 p^{\prime}+1$ and $q=2 q^{\prime}+1$, sets $N=p q, m=p^{\prime} q^{\prime}$, selects a generator $G$ of the group of signed quadratic residues $\mathbb{Q R}_{N}^{+}$, and outputs $\left(\left[\mathbb{Q R}_{N}^{+}\right], G, m\right)$. Algorithm Samp SQR $_{\text {, }}$ on input $\left(\left[\mathbb{Q R}_{N}^{+}\right], G, m\right)$, selects a random $x \in[0 ; m-1]$, sets $X=G^{x}, \tau_{x}=\xi(x)$, and outputs $\left(X, x, \tau_{x}\right)$. The algorithm Solve $\mathrm{SQR}_{\mathrm{R}}$, on input $\left(\left[\mathbb{Q R}_{N}^{+}\right], G ; X, Y, Z ; \tau_{x}\right)$, first checks that the trapdoor is correct by verifying whether $G^{\tau_{x}}=X^{2}$ (it outputs $\perp$ if this does not hold), and outputs 1 iff $Y^{\tau_{x}}=Z^{2}$. We now formally prove that this constitutes a static TDDH group under appropriate assumptions (the proof of property $i$ ) is reminiscent of the one of Theorem 3.2 in [31]).

Theorem 3. Under the factoring assumption (for the product of safe primes) and the DDH assumption for $\mathbb{Q R}_{N}^{+}, \mathcal{S} \mathcal{T} \mathcal{D} \mathcal{D} \mathcal{H}_{\mathrm{SQR}}$ is a static TDDH group with perfect soundness.

Proof. Deferred to the full version of the paper [45] for reasons of space.

\subsection{Relation to the Strong Diffie-Hellman Problem}

We note that in a static TDDH group with perfect soundness, the Strong DiffieHellman (SDH) problem [1] is always hard 8 The SDH problem is to compute $\mathrm{CDH}_{G}(X, Y)$ given $X, Y \in\langle G\rangle$, and being granted access to a static DDH oracle which on input $\left(Y^{\prime}, Z^{\prime}\right) \in \mathbb{G}^{2}$ outputs 1 iff $\left(X, Y^{\prime}, Z^{\prime}\right) \in \mathcal{D H}_{G}$. Clearly, an adversary $\mathcal{A}$ breaking the $\mathrm{SDH}$ problem can be turned into an adversary $\mathcal{B}$ breaking property $i$ ) of the static TDDH group ( $\mathcal{B}$ can answer queries of $\mathcal{A}$ to the static DDH oracle thanks to the trapdoor $\tau_{x}$ it is given as input). Applying this observation to $\mathcal{S} \mathcal{T} \mathcal{D} \mathcal{D} \mathcal{H}_{\mathrm{SQR}}$, we recover Theorem 3.2 of [31] which states that $\mathrm{SDH}$ is hard in $\mathbb{Q} \mathbb{R}_{N}^{+}$under the factoring assumption. Hence, the concept of static TDDH group allows to cast the result of [31] in a more general framework. In particular, Theorem 2 directly implies that under the RSA assumption, the SDH problem is hard in $\mathbb{J}_{N}$, which complements the result of [31] As an immediate consequence of the results of [115], we obtain that Hybrid ElGamal encryption over $\mathbb{J}_{N}$ is IND-CCA2-secure in the ROM under the RSA assumption.

\section{Convertible Undeniable Signatures}

\subsection{Background on Undeniable Signatures}

In this section, we show how TDDH groups can be used to build simple and natural undeniable signature schemes with attractive properties such as universal convertibility and delegation. Undeniable signatures, introduced by Chaum

\footnotetext{
${ }^{8}$ More precisely, the SDH problem is hard for the group generator which only outputs $([\mathbb{G}], G)$.

${ }^{9}$ Note that, by inspection of the proof of property $\left.i i\right)$, this result holds in fact for all RSA moduli $N$ such that $\mathbb{J}_{N}$ is cyclic, not only the product of safe primes.
} 
and van Antwerpen [12, are signatures that cannot be universally verified: confirmation (or disavowal) of a signature requires the cooperation of the signer (however a signer cannot deny the validity of a correct signature, hence the name undeniable). Later, Boyar et al. [5] proposed the refined notion of convertible undeniable signature (CUS) scheme, where a mechanism allows the signer to selectively or globally transform undeniable signatures into self-authenticating signatures. The particular scheme proposed in [5] was later broken in [36. Subsequently, schemes based on usual signatures such as ElGamal [17], Schnorr [37, and RSA [26]24]23] were proposed.

We first recall the basic Chaum and van Antwerpen undeniable signature scheme [12] (in its Full Domain Hash version [4139]). Let $\mathbb{G}$ be a group, $\mathbb{G}^{\prime}$ be a cyclic and efficiently recognizable subgroup of $\mathbb{G}, G$ be a (certified) generator of $\mathbb{G}^{\prime}$, and $\boldsymbol{H}:\{0,1\}^{*} \rightarrow \mathbb{G}^{\prime}$ be a hash function (modeled as a random oracle in security proofs). Assume the DDH problem is hard for $\mathbb{G}^{\prime}$. The secret and public keys of a user are $x \in \mathbb{Z}_{\left|\mathbb{G}^{\prime}\right|+}$ and $X=G^{x}$ respectively. To sign a message $\mu \in\{0,1\}^{*}$, the signer computes $M=\boldsymbol{H}(\mu) \in \mathbb{G}^{\prime}$, and $S=M^{x}$. The signature is $S$. A signature $S$ on $\mu$ is valid iff $(X, \boldsymbol{H}(\mu), S)$ is a valid DH tuple (with respect to $G)$. Since we assumed that the DDH problem is hard, checking the validity of a signature cannot be done without knowledge of $x 10$ Hence, the signer must cooperate with the verifier in order to confirm or disavow a purported signature. The confirmation protocol is a proof that $(X, \boldsymbol{H}(\mu), S) \in \mathcal{D H}_{G}$ (i.e. a proof of EDL since $G$ is guaranteed to be a generator of $\mathbb{G}^{\prime}$ ), whereas the disavowal protocol is a proof that $(X, \boldsymbol{H}(\mu), S) \notin \mathcal{D} \mathcal{H}_{G}$ (i.e. a proof of IDL). The security of this scheme (depending on which type of EDL and IDL proofs are used) has been studied in 41/34/39/33.

The idea to allow efficient universal conversion of signatures is simply to use a Chaum and van Antwerpen undeniable signature with a (static or not) TDDH group, and to use the trapdoor to delegate the ability to verify undeniable signatures and to universally convert them. In the following, we describe the construction using static TDDH groups since the instantiations using constructions of Sections 4.2 and 4.3 are particularly interesting.

\subsection{Construction of a CUS scheme from a Static TDDH Group}

Let $\mathcal{S T D D H}=($ Gen, Samp, Solve $)$ be a static TDDH group with perfect soundness. For this part, we assume that Gen outputs a tuple $([\mathbb{G}], G, \tau)$ such that $\mathbb{G}$ is cyclic and efficiently recognizable, and $G$ is a generator of $\mathbb{G}$. This assumption

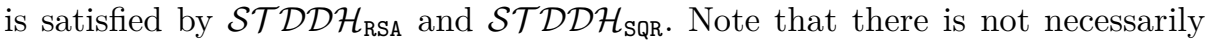
an efficient way to check that $G$ is indeed a generator; we come back on this issue later. We construct a CUS scheme $\mathcal{C U S}$ as follows (see the full version of the paper [45] for a more formal description). To construct his public/secret key pair, the signer runs $\operatorname{Gen}\left(1^{k}\right)$ to obtain $([\mathbb{G}], G, \tau)$ and then $\operatorname{Samp}([\mathbb{G}], G, \tau)$ to

${ }^{10}$ When the DDH problem is easy in $\mathbb{G}^{\prime}$, signatures can be universally verified. For example, using bilinear groups (where a pairing can be used to solve the DDH problem), one obtains the Boneh-Lynn-Shacham signature scheme 4]. 
obtain $\left(X, x, \tau_{x}\right)$. It also selects a hash function $\boldsymbol{H}:\{0,1\}^{*} \rightarrow \mathbb{G}$. The public key of the signer is $\mathrm{pk}=([\mathbb{G}], G, X, \boldsymbol{H})$ and its secret key is sk $=\left(x, \tau_{x}\right)$. To sign a message $\mu \in\{0,1\}^{*}$, the signer computes $M=\boldsymbol{H}(\mu)$, and $S=M^{x}$. The signature is $S$. The signer can confirm or disavow a signature by running a proof of EDL or IDL respectively with the verifier. To individually convert a signature, the signer produces a NIZK proof of EDL (using an independent hash function $\boldsymbol{H}_{\mathrm{FS}}$ to apply the Fiat-Shamir transform). To universally convert signatures, the signer releases $\tau_{x}$ as universal receipt. A signature $S$ for message $\mu$ can then be verified by running $\operatorname{Solve}\left([\mathbb{G}], G ; X, \boldsymbol{H}(\mu), S ; \tau_{x}\right)$.

Informally, the two main security properties of a CUS scheme (beside soundness of the confirmation and disavowal protocols) are (see the full version of the paper [45] for details):

- security against existential forgery under chosen-message attacks (EF-CMAsecurity): any PPT attacker, given the receipt for universal verification $\tau_{x}$, and with access to a signing oracle, can forge a new signature with only negligible probability (note that access to confirmation or disavowal oracles is unnecessary here since the adversary is given the universal receipt $\tau_{x}$ for checking signatures);

- invisibility under chosen-message attacks (INV-CMA-security): any PPT adversary can distinguish a valid signature for a message of its choice from a string sampled uniformly at random from the signature space with only negligible probability. The adversary is granted access to the signing oracle, the confirmation and disavowal protocols, and the individual signature conversion oracle (with the restriction that they cannot be queried on the challenge message).

We stress that formalizing the invisibility notion is quite subtle (many variations appear in the literature $1317 / 8,23]$ ), and that the exact property that is achieved is dependent on the nature of the confirmation and disavowal protocols [39 33].

Theorem 4. When instantiated with a static TDDH group with perfect soundness, and when the confirmation and disavowal protocols are zero-knowledge, the CUS scheme described above is EF-CMA-secure and INV-CMA-secure in the $R O M$ (for $\boldsymbol{H}$ and $\boldsymbol{H}_{\mathrm{FS}}$ ).

Proof. Deferred to the full version of the paper [45] for reasons of space.

Delegation. The ability to verify (confirm or disavow) and convert (either individually or universally) signatures can easily be delegated to a semi-trusted party by simply giving him the trapdoor $\tau_{x}$. Since the CDH problem remains hard even with the trapdoor, the third party cannot forge signatures on behalf of the signer. It can however prove in zero-knowledge whether a signature is valid or invalid (since it knows the witness $\tau_{x}$ for this). We avoid using the term designated confirmer signatures [10] here since this usually refers to schemes (mostly following the "encryption of a signature" paradigm 40 8]) where the signer can create designated confirmer undeniable signatures without having beforehand to transmit some secret information to the confirmer (in our case the trapdoor $\tau_{x}$ ). 
Instantiation with $\mathcal{S T} \mathcal{D} \mathcal{D} \mathcal{H}_{\mathrm{RSA}}$ and $\mathcal{S} \mathcal{T} \mathcal{D} \mathcal{D} \mathcal{H}_{\mathrm{SQR}}$. The CUS scheme described above can be instantiated with the two static TDDH groups described in Sections 4.2 and 4.3 The schemes obtained this way are similar respectively to the scheme of Gennaro, Rabin, and Krawczyk [26] and Galbraith and Mao [23], with important distinctions though. Both schemes work over $\mathbb{Z}_{N}^{*}$, but without explicitly restricting in which subgroup. As a consequence, they cannot be exactly seen as an instantiation of the Chaum and van Antwerpen scheme, and specific confirmation and disavowal protocols were therefore proposed for them (see also [24]). On the contrary, our schemes are strict instantiations of the Chaum and van Antwerpen scheme, and in particular the confirmation and disavowal protocols can use zero-knowledge proofs of EDL and IDL derived from the HVZK protocols described in the full version of the paper [45. This is conceptually simpler and more efficient (especially for the disavowal protocol).

Certifying Signers Public Keys. Correct key generation is of primary importance in factoring-based undeniable signatures, since a cheating signer may generate its secret/public key in a different way than the one expected by verifiers, which may enable him to confirm invalid signatures or disavow valid ones (see [24]). Hence, the signer, when registering his public key, must prove to the certification authority $(\mathrm{CA})$ that it was generated according to the specification of the static TDDH group generator. We now discuss this issue with respect to $\mathcal{S T} \mathcal{D} \mathcal{D} \mathcal{H}_{\mathrm{RSA}}$ and $\mathcal{S} \mathcal{T} \mathcal{D} \mathcal{D} \mathcal{H}_{\mathrm{SQR}}$. For both schemes, the signer must first prove to the CA that its modulus $N$ is the product of two safe primes. A zero-knowledge protocol for this was proposed by Camenish and Michels [7]. Though expensive, this protocol must be run only once at key registration time. Then, the signer must prove that $G$ is indeed a generator of either $\mathbb{J}_{N}$ or $\mathbb{Q R}_{N}^{+}$. The situation is slightly different in the two cases. Denote $N=p q$ with $p=2 p^{\prime}+1$ and $q=2 q^{\prime}+1$. When $p$ and $q$ are safe primes, then an integer $g \in \mathbb{Z}_{N}^{*}$ such that $g^{2} \neq 1 \bmod N$ and $\operatorname{gcd}\left(g^{2}-1, N\right)=1$ necessarily has order in $\left\{p^{\prime} q^{\prime}, 2 p^{\prime} q^{\prime}\right\}$ [26. Lemma 1]. Hence, an ad-hoc solution for ensuring that the element $G$ provided by the signer is a generator of the intended group is as follows. Restrict the scheme to moduli $N$ such that $N \equiv 1 \bmod 8$ and fix $g_{0}=2$ so that $g_{0} \in \mathbb{J}_{N}$. Since an element $g \in \mathbb{Q R}_{N}^{+}$generates $\mathbb{Q R}_{N}^{+}$exactly when $g$ has multiplicative order modulo $N$ in $\left\{p^{\prime} q^{\prime}, 2 p^{\prime} q^{\prime}\right\}$, we see by the previous remark that $g_{0}$ is always a generator of $\mathbb{Q R}_{N}^{+}$. Hence, when using $\mathcal{S} \mathcal{T} \mathcal{D} D \mathcal{H}_{\mathrm{SQR}}$, we can impose to the signer to always use $G=g_{0}$. Things are a bit more complicated when using $\mathcal{S T D D} \mathcal{H}_{\mathrm{RSA}}$, since for an element $g \in \mathbb{Z}_{N}^{*}$ with order in $\left\{p^{\prime} q^{\prime}, 2 p^{\prime} q^{\prime}\right\}$ to generate $\mathbb{J}_{N}$, one has to check that it is a quadratic non-residue. What we propose for this is that the signer proves in zero-knowledge to the CA whether $g_{0} \in \mathbb{Q R}_{N}$ or not [30. If it is in $\mathbb{Q R}_{N}$, then the signer tries with $g_{0}+1, g_{0}+2$, etc. until a quadratic non-residue in $\mathbb{J}_{N}$ is found. The signer then has to use $G=g_{0}+i$ for the smallest $i \geq 0$ such that $g_{0}+i \in \mathbb{J}_{N} \backslash \mathbb{Q R}_{N}$.

As a matter of fact, there seems to be no reason to instantiate the CUS scheme with $\mathcal{S} \mathcal{T} \mathcal{D} \mathcal{D} \mathcal{H}_{\mathrm{RSA}}$ rather than $\mathcal{S} \mathcal{T} \mathcal{D} \mathcal{D} \mathcal{H}_{\mathrm{SQR}}$ since both schemes are almost identical, except that the key registration step is simpler for $\mathcal{S} \mathcal{T} \mathcal{D D} \mathcal{H}_{\mathrm{SQR}}$. 


\section{References}

1. Abdalla, M., Bellare, M., Rogaway, P.: The Oracle Diffie-Hellman Assumptions and an Analysis of DHIES. In: Naccache, D. (ed.) CT-RSA 2001. LNCS, vol. 2020, pp. 143-158. Springer, Heidelberg (2001)

2. Boneh, D.: The Decision Diffie-Hellman Problem. In: Buhler, J.P. (ed.) ANTS 1998. LNCS, vol. 1423, pp. 48-63. Springer, Heidelberg (1998)

3. Boneh, D., Franklin, M.: Identity-Based Encryption from the Weil Pairing. In: Kilian, J. (ed.) CRYPTO 2001. LNCS, vol. 2139, pp. 213-229. Springer, Heidelberg (2001)

4. Boneh, D., Lynn, B., Shacham, H.: Short Signatures from the Weil Pairing. Journal of Cryptology 17(4), 297-319 (2004)

5. Boyar, J., Chaum, D., Damgård, I., Pedersen, T.P.: Convertible Undeniable Signatures. In: Menezes, A., Vanstone, S.A. (eds.) CRYPTO 1990. LNCS, vol. 537, pp. 189-205. Springer, Heidelberg (1991)

6. Bresson, E., Catalano, D., Pointcheval, D.: A Simple Public-Key Cryptosystem with a Double Trapdoor Decryption Mechanism and Its Applications. In: Laih, C.-S. (ed.) ASIACRYPT 2003. LNCS, vol. 2894, pp. 37-54. Springer, Heidelberg (2003)

7. Camenisch, J., Michels, M.: Proving in Zero-Knowledge that a Number Is the Product of Two Safe Primes. In: Stern, J. (ed.) EUROCRYPT 1999. LNCS, vol. 1592, pp. 107-122. Springer, Heidelberg (1999)

8. Camenisch, J., Michels, M.: Confirmer Signature Schemes Secure against Adaptive Adversaries (Extended Abstract). In: Preneel, B. (ed.) EUROCRYPT 2000. LNCS, vol. 1807, pp. 243-258. Springer, Heidelberg (2000)

9. Camenisch, J., Shoup, V.: Practical Verifiable Encryption and Decryption of Discrete Logarithms. In: Boneh, D. (ed.) CRYPTO 2003. LNCS, vol. 2729, pp. 126144. Springer, Heidelberg (2003)

10. Chaum, D.: Designated Confirmer Signatures. In: De Santis, A. (ed.) EUROCRYPT 1994. LNCS, vol. 950, pp. 86-91. Springer, Heidelberg (1995)

11. Chaum, D., Pedersen, T.P.: Wallet Databases with Observers. In: Brickell, E.F. (ed.) CRYPTO 1992. LNCS, vol. 740, pp. 89-105. Springer, Heidelberg (1993)

12. Chaum, D., van Antwerpen, H.: Undeniable Signatures. In: Brassard, G. (ed.) CRYPTO 1989. LNCS, vol. 435, pp. 212-216. Springer, Heidelberg (1990)

13. Chaum, D., van Heijst, E., Pfitzmann, B.: Cryptographically Strong Undeniable Signatures, Unconditionally Secure for the Signer. In: Feigenbaum, J. (ed.) CRYPTO 1991. LNCS, vol. 576, pp. 470-484. Springer, Heidelberg (1992)

14. Cramer, R., Damgård, I., MacKenzie, P.D.: Efficient Zero-Knowledge Proofs of Knowledge without Intractability Assumptions. In: Imai, H., Zheng, Y. (eds.) PKC 2000. LNCS, vol. 1751, pp. 354-373. Springer, Heidelberg (2000)

15. Cramer, R., Shoup, V.: Design and Analysis of Practical Public-Key Encryption Schemes Secure against Adaptive Chosen Ciphertext Attack. SIAM Journal on Computing 33(1), 167-226 (2003)

16. Damgård, I.: Efficient Concurrent Zero-Knowledge in the Auxiliary String Model. In: Preneel, B. (ed.) EUROCRYPT 2000. LNCS, vol. 1807, pp. 418-430. Springer, Heidelberg (2000)

17. Damgård, I., Pedersen, T.P.: New Convertible Undeniable Signature Schemes. In: Maurer, U.M. (ed.) EUROCRYPT 1996. LNCS, vol. 1070, pp. 372-386. Springer, Heidelberg (1996) 
18. Dent, A.W., Galbraith, S.D.: Hidden Pairings and Trapdoor DDH Groups. In: Hess, F., Pauli, S., Pohst, M. (eds.) ANTS 2006. LNCS, vol. 4076, pp. 436-451. Springer, Heidelberg (2006)

19. Desmedt, Y., Frankel, Y.: Threshold Cryptosystems. In: Brassard, G. (ed.) CRYPTO 1989. LNCS, vol. 435, pp. 307-315. Springer, Heidelberg (1990)

20. Fiat, A., Shamir, A.: How to Prove Yourself: Practical Solutions to Identification and Signature Problems. In: Odlyzko, A.M. (ed.) CRYPTO 1986. LNCS, vol. 263, pp. 186-194. Springer, Heidelberg (1987)

21. Frey, G.: How to disguise an elliptic curve (Weil descent). In: Elliptic Curve Cryptography - ECC 1998 (1998), http://cacr.uwaterloo.ca/conferences/1998/ecc98/frey.ps

22. Frey, G., Müller, M., Rück, H.-G.: The Tate pairing and the discrete logarithm applied to elliptic curve cryptosystems. IEEE Transactions on Information Theory 45(5), 1717-1719 (1999)

23. Galbraith, S.D., Mao, W.: Invisibility and Anonymity of Undeniable and Confirmer Signatures. In: Joye, M. (ed.) CT-RSA 2003. LNCS, vol. 2612, pp. 80-97. Springer, Heidelberg (2003)

24. Galbraith, S.D., Mao, W., Paterson, K.G.: RSA-Based Undeniable Signatures for General Moduli. In: Preneel, B. (ed.) CT-RSA 2002. LNCS, vol. 2271, pp. 200-217. Springer, Heidelberg (2002)

25. Gennaro, R.: Multi-trapdoor Commitments and Their Applications to Proofs of Knowledge Secure Under Concurrent Man-in-the-Middle Attacks. In: Franklin, M. (ed.) CRYPTO 2004. LNCS, vol. 3152, pp. 220-236. Springer, Heidelberg (2004)

26. Gennaro, R., Rabin, T., Krawczyk, H.: RSA-Based Undeniable Signatures. Journal of Cryptology 13(4), 397-416 (2000)

27. Girault, M.: An Identity-Based Identification Scheme Based on Discrete Logarithms Modulo a Composite Number. In: Damgård, I.B. (ed.) EUROCRYPT 1990. LNCS, vol. 473, pp. 481-486. Springer, Heidelberg (1991)

28. Girault, M.: Self-Certified Public Keys. In: Davies, D.W. (ed.) EUROCRYPT 1991. LNCS, vol. 547, pp. 490-497. Springer, Heidelberg (1991)

29. Goldreich, O., Sahai, A., Vadhan, S.P.: Honest-Verifier Statistical Zero-Knowledge Equals General Statistical Zero-Knowledge. In: Vitter, J.S. (ed.) Symposium on the Theory of Computing - STOC 1998, pp. 399-408. ACM (1998)

30. Goldwasser, S., Micali, S., Rackoff, C.: The Knowledge Complexity of Interactive Proof Systems. SIAM Journal on Computing 18(1), 186-208 (1989)

31. Hofheinz, D., Kiltz, E.: The Group of Signed Quadratic Residues and Applications. In: Halevi, S. (ed.) CRYPTO 2009. LNCS, vol. 5677, pp. 637-653. Springer, Heidelberg (2009)

32. Joux, A.: A One Round Protocol for Tripartite Diffie-Hellman. In: Bosma, W. (ed.) ANTS 2000. LNCS, vol. 1838, pp. 385-394. Springer, Heidelberg (2000)

33. Kurosawa, K., Furukawa, J.: Universally Composable Undeniable Signature. In: Aceto, L., Damgård, I., Goldberg, L.A., Halldórsson, M.M., Ingólfsdóttir, A., Walukiewicz, I. (eds.) ICALP 2008, Part II. LNCS, vol. 5126, pp. 524-535. Springer, Heidelberg (2008)

34. Kurosawa, K., Heng, S.-H.: 3-Move Undeniable Signature Scheme. In: Cramer, R. (ed.) EUROCRYPT 2005. LNCS, vol. 3494, pp. 181-197. Springer, Heidelberg (2005)

35. Menezes, A., Okamoto, T., Vanstone, S.A.: Reducing elliptic curve logarithms to logarithms in a finite field. IEEE Transactions on Information Theory 39(5), 16391646 (1993) 
36. Michels, M., Petersen, H., Horster, P.: Breaking and Repairing a Convertible Undeniable Signature Scheme. In: Gong, L., Stearn, J. (eds.) ACM Conference on Computer and Communications Security - CCS 1996, pp. 148-152. ACM (1996)

37. Michels, M., Stadler, M.: Efficient Convertible Undeniable Signature Schemes. In: Selected Areas in Cryptography - SAC 1997, pp. 231-244 (1997)

38. Morales, D.J.M.: An attack on disguised elliptic curves. Journal of Mathematical Cryptology 2(1), 1-8 (2008), http://eprint.iacr.org/2006/469.pdf

39. Ogata, W., Kurosawa, K., Heng, S.-H.: The Security of the FDH Variant of Chaum's Undeniable Signature Scheme. In: Vaudenay, S. (ed.) PKC 2005. LNCS, vol. 3386, pp. 328-345. Springer, Heidelberg (2005)

40. Okamoto, T.: Designated Confirmer Signatures and Public-Key Encryption Are Equivalent. In: Desmedt, Y.G. (ed.) CRYPTO 1994. LNCS, vol. 839, pp. 61-74. Springer, Heidelberg (1994)

41. Okamoto, T., Pointcheval, D.: The Gap-Problems: A New Class of Problems for the Security of Cryptographic Schemes. In: Kim, K. (ed.) PKC 2001. LNCS, vol. 1992, pp. 104-118. Springer, Heidelberg (2001)

42. Paillier, P.: Public-Key Cryptosystems Based on Composite Degree Residuosity Classes. In: Stern, J. (ed.) EUROCRYPT 1999. LNCS, vol. 1592, pp. 223-238. Springer, Heidelberg (1999)

43. Prabhakaran, M., Xue, R.: Statistically Hiding Sets. In: Fischlin, M. (ed.) CT-RSA 2009. LNCS, vol. 5473, pp. 100-116. Springer, Heidelberg (2009)

44. Schnorr, C.-P.: Efficient Signature Generation by Smart Cards. Journal of Cryptology 4(3), 161-174 (1991)

45. Seurin, Y.: New Constructions and Applications of Trapdoor DDH Groups. Full version of this paper. Available from the author or from http://eprint.iacr.org

46. Tsiounis, Y., Yung, M.: On the Security of ElGamal Based Encryption. In: Imai, H., Zheng, Y. (eds.) PKC 1998. LNCS, vol. 1431, pp. 117-134. Springer, Heidelberg (1998) 